\title{
Individual differences in working memory capacity predict visual attention allocation
}

\author{
M. KATHRYN BLECKLEY and FRANCIS T. DURSO \\ Texas Tech University, Lubbock, Texas \\ JERRY M. CRUTCHFIELD \\ Boeing Company, Seattle, Washington \\ RANDALL W. ENGLE \\ Georgia Institute of Technology, Atlanta, Georgia \\ and \\ MAYA M. KHANNA \\ University of Michigan, Ann Arbor, Michigan
}

\begin{abstract}
To the extent that individual differences in working memory capacity (WMC) reflect differences in attention (Baddeley, 1993; Engle, Kane, \& Tuholski, 1999), differences in WMC should predict performance on visual attention tasks. Individuals who scored in the upper and lower quartiles on the OSPAN working memory test performed a modification of Egly and Homa's (1984) selective attention task. In this task, the participants identified a central letter and localized a displaced letter flashed somewhere on one of three concentric rings. When the displaced letter occurred closer to fixation than the cue implied, high-WMC, but not low-WMC, individuals showed a cost in the letter localization task. This suggests that low-WMC participants allocated attention as a spotlight, whereas those with high WMC showed flexible allocation.
\end{abstract}

Although working memory (WM) has been at the core of discussions about both human and animal cognition in recent years, there are many views about the nature of the limits on temporarily active memory. A commonly held view, based on Miller's (1956) classic paper, has characterized limitations in terms of the number of items or chunks that can be temporarily active. This view arose naturally from the tasks that have been used to measure that capacity. Reading span (Daneman \& Carpenter, 1980) and operation-word span (Turner \& Engle, 1989) are scored by the number of words that are recalled correctly. This number-of-bins viewpoint has been corroborated by the ability of these tasks to predict such wide-ranging abilities as note taking (Kiewra \& Benton, 1988), bridge playing (Clarkson-Smith \& Hartley, 1990), computer language learning (Shute, 1991), and reasoning (Kyllonen \& Christal, 1990). If one has more bins available, one is able

Data for this study were collected at the University of Oklahoma, where M.M.K.'s participation was funded by NSF REU Grant SES9820689 to F.T.D. The authors thank Michael J. Kane, Veronica Dark, Nelson Cowan, Sowon Hahn, and an anonymous reviewer for their valuable comments during the preparation of this manuscript. Correspondence concerning this article should be directed to M. K. Bleckley, Department of Psychology, Texas Tech University, P. O. Box 42051, Lubbock, TX 79409-2051 (e-mail: kate.bleckley@ ttu.edu).

Note-This article was accepted by the previous editorial team, while John T. Wixted was editor. to take more detailed notes (more bins, more details). More bins also allow one to remember longer bidding sequences or to remember the composition of more tricks. When a new language is learned, more bins mean that there are more associations available at one time. In addition, more bins allow more parts of the puzzle to remain in an active state for analysis and, ultimately, solution.

The number-of-bins view has appeared in a modified form in Cowan's $(1999,2001)$ recent work. Cowan's (2001) view holds that the limit on central capacity is applicable to the focus of attention. This limit on fundamental capacity is detected when the chunks are held in the focus of attention at one time; so, it is the focus of attention that appears to be limited to approximately four chunks.

Alternative views have been based on Baddeley and Hitch's (1994) contention that WM consists of an attentionbased central executive that makes use of a variety of buffers, which vary in representational format, in order to maintain information (e.g., speech, visual/spatial, etc.). Engle and his colleagues (Engle, Tuholski, Laughlin, \& Conway, 1999; Turner \& Engle, 1989) have used individual differences in WM capacity (WMC) to study the nature of these limitations. They have argued that the relationship between measures of WMC and higher level cognitive tasks reflects differences in the domain-free ability to maintain task-relevant context-specific information in a highly active and easily retrievable state in 
the face of distracting information. This is essentially an attentional mechanism.

The assertion that this construct is attentional and domain free has received support from a variety of experiments in which individuals measured to be high and low in WMC have been used. Kane, Bleckley, Conway, and Engle (2001) found that WMC differences predicted performance on the antisaccade task, in which participants must suppress an automatic saccade to a warning signal in order to identify a stimulus on the opposite side of the visual field. Their experiment had two conditions: prosaccade, in which the stimuli (letters) to be identified occurred in the same location as the warning signal, and antisaccade, in which the stimuli occurred on the opposite side of the visual field from the cue. In the prosaccade condition, there were no differences in response time or errors between the groups; however, in the antisaccade condition, low-span individuals were slower to identify the target letter, suggesting that they had difficulty suppressing saccades toward the cue. Their second experiment measured eye movements. Kane et al. found that low-span individuals were slower than high-span individuals to initiate a saccade after the cue had occurred and made significantly more saccades toward the cue than did high-span individuals in the antisaccade condition. These differences remained across 10 blocks of trials. Again, if WMC is conceived of as the number of items that can be held in memory, one would not predict group differences in the ability to suppress saccades toward the cue, but by reconceptualizing WMC as the ability to control attention, Kane et al. predicted and confirmed an impact of WM on attentional processing.

Finally, Conway, Cowan, and Bunting (2001) found that WMC is important in focusing on relevant auditory information and suppressing irrelevant information. Using Moray's (1959) dichotic listening task, in which the participant's name is presented on the unattended channel at the 4th min of the shadowing task, they found that only $20 \%$ of the high-span participants heard their name-often referred to as the cocktail party phenomenon-whereas $62 \%$ of the low-span participants reported having heard their name. Again, WMC predicted group differences in the ability to control attention. The unifying theme of these studies is that controlled attention is pivotal in the performance of the tasks and that measures of WMC reflect that.

Proponents of the controlled attention view of WM contend that differences in WMC influence performance on tasks that require suppression of information irrelevant to the task. This leads to the prediction that differences in WMC would result in differences in the allocation of visual attention. In studies of visual processing, attention acts not only to facilitate processing of selected information (Posner, Snyder, \& Davidson, 1980), but also to suppress distractor information. Cepeda, Cave, Bichot, and Kim (1998) found that attention could operate by inhibiting distractor information, as well as by enhancing target information. They used a dual-task probe tech- nique wherein participants first searched for targets of a certain color among distractors of another color. Then the participants responded to probes at locations previously occupied by the target, a distractor, or a neutral location. Cepeda et al. found that probe reaction times were shorter for target locations, when compared with neutral locations, supporting Posner et al.'s assertion that attention facilitates processing. However, Cepeda et al. also found that RTs to distractor locations were longer than RTs to neutral locations, suggesting that attention also serves to inhibit distractor locations.

If WMC influences the allocation of visual attention, one might anticipate a difference in the way in which high$\mathrm{WM}$ and low-WM individuals allocate attention. One of the most well-known models of allocation of visual attention is Posner et al.'s (1980) spotlightmodel. In this model, the focus of attention is mobile, and stimuli within its area are processed. LaBerge (1983) modified the spotlight model to account for data showing a gradual decline in attentional acuity as the distance from the center of fixation increased (a gradient model). These and other spotlight, gradient, and zoom-lens models of visual attention assume a contiguous area of attention. However, Egly and Homa (1984), among others (e.g., Awh \& Pashler, 2000; Castiello \& Umiltà, 1992; Kramer \& Hahn, 1995; see Cave \& Bichot, 1999, for a review), found that visual attention can sometimes be allocated to discontiguous locations. Discontiguous allocation is said to have taken place when the target was not detected by the participant even though it occurred between two cued locations.

On the basis of these findings, we tested the prediction of the controlled attention view that WMC, now reconceptualized as controlled attention, would have an effect on the allocation of visual attention. We predicted that high WM span individuals would be able to attend selectively to the areas of the visual field that were important to the task while ignoring or inhibiting other areas, whereas low WM span individuals would be less able to allocate their attention flexibly. It may be the case that the spotlight metaphor of a continuous, if graded, allocation of attention, originally proposed by Posner et al. (1980), would provide a good model for the allocation of attention in low WM span individuals but might miss the flexible nature of attention allocation in high WM span individuals.

We tested this prediction by borrowing from Egly and Homa (1984), who developed a procedure by which to distinguish between attention allocated as a spotlight and a more flexible allocation of visual attention. They showed that the students in their study could allocate attention simultaneously to two different locations: to a central location and to one of three concentric rings surrounding the center (see Figure 1). The students were asked to identify the briefly presented center letter and to localize a displaced letter that was simultaneously presented on one of the rings. When the participants were given a valid cue indicating on which ring the letter would appear, localization accuracy, of course, im- 


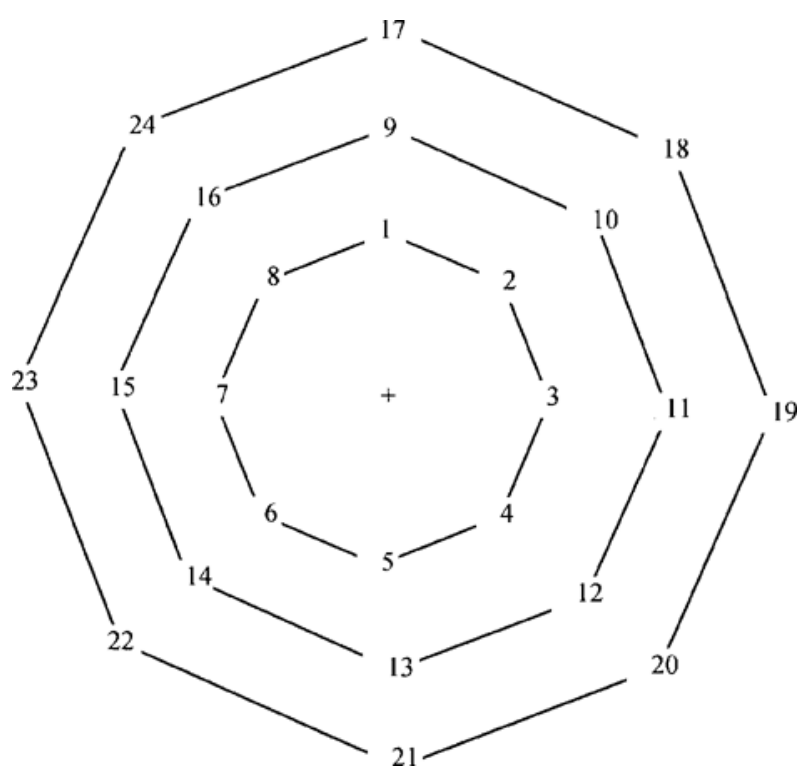

Figure 1. The spiderweb grid showing the three rings.

proved. Of prime interest is what happened when the wrong ring was cued. When the letter appeared on a ring outside of the cued ring, performance suffered. This finding is consistent with either a spotlight model or any view of flexible allocation, because the target occurred outside of the focus of attention. More important is the finding that when the letter appeared on a ring inside of the cued ring, performance also suffered. Because accuracy decreased when the target appeared either outside the cued ring or inside the cued ring, Egly and Homa ruled out a simple spotlight. A spotlight would have been suggested had performance remained high when the target appeared at any location between the center and the cued ring.

In the present study, we attempted to replicate Egly and Homa's (1984) finding and to determine whether it would apply to both high and low WM span individuals. The controlled attention view of WM led to the hypothesis that discontiguous allocation would be found for high, but not for low, WM spans. If the groups could be equated on validly cued trials, this prediction would include the unusual expectation that low WM span individuals' performance would exceed high WM span performance on invalidly cued trials in which the letter occurred closer to fixation than the cue indicated.

\section{METHOD}

\section{Working Memory Capacity}

The participants were screened for their WM capacity, using the operation-word-span task (OSPAN), in which they verified series of simple mathematical operations while they attempted to remember a list of unrelated words (Turner \& Engle, 1989). The participants read aloud each equation, stated whether it was correct or not, and spoke aloud the word. The experimenter then caused the next equation-word pair to be presented. "Is $(2 \times 3)+1=4$ ? aunt" is a sample equation-word pair. At the end of each set of equation-word pairs, the participants wrote the words recalled from the set. Points were scored only for perfect serial recall. The participants were tested on three lists of each length (from two to six operation-word pairs), so possible total scores ranged from 0 to 60 . The list size order, operations, and words were randomized for each participant.

Twenty participants, 10 of whom scored in the lowest quartile (span "10) and 10 of whom scored in the highest quartile (span $\geq 18$ ), were recruited for the visual attention study. Quartiles were based on a database comprising results from several studies and universities (see, e.g., Conway et al., 2001; Kane et al., 2001). Low WM span scores ranged from 4 to 10 , with a mean of 8.4. High WM span scores ranged from 21 to 49 , with a mean of 30.3 .

\section{Visual Attention Allocation Task}

We used the methods from Egly and Homa's (1984) Experiment 3 , with the exceptions that stimuli were presented via a computer monitor, instead of a tachistoscope, and location cues (i.e., close, medium, or distant) were presented as words on the monitor, rather than by being spoken. The participants were instructed to identify a letter flashed briefly at the center of fixation and to locate a letter presented on one of three concentric octagons, spaced at intervals of $1^{\circ}$ of visual angle (see Figure 1). Experimental stimuli consisted of a pair of letters, one presented at the center of the grid and the second presented at one of the 24 locations on the octagons. The pairs of letters were selected from the set of nine letters used by Egly and Homa (C, O, Q, F, L, T, V, X, and Y). The displaced letter appeared at $1^{\circ}$ of visual angle from the center letter one third of the time, at $2^{\circ}$ of visual angle one third of the time, and at $3^{\circ}$ of visual angle one third of the time. Trials could be uncued, with no indication of where the displaced letter would occur, validly cued, with the cue word indicating on which ring the displaced letter would occur, or invalidly cued, with the cue word indicating where the displaced letter would not occur.

The participants completed one session on each of 3 consecutive days. On the 1st day, the session began with orientation to the task. The participants practiced the task with 10 uncued trials, then 5 validly cued trials, and finally 3 invalidly cued trials. The participants then received the experimental instructions taken from Egly and Homa (1984). The experimental instructions emphasized accuracy, with the central letter receiving more emphasis than the displaced letter. Because the experimenter entered the participants' responses, no reaction time data were collected.

These instructions were followed by a block of 10 trials, containing both cued and uncued trials. All the participants started with a display time of $50 \mathrm{msec}$ (three refresh cycles); then, on the basis of the participant's accuracy in identifying the center letter, the display time was adjusted. If the participant incorrectly identified the center letter on $20 \%$ or more of the practice trials, the display duration was increased by one refresh cycle to $67 \mathrm{msec}$. If they were incorrect on $10 \%$ or fewer of the practice trials, their display duration was decreased by one refresh cycle (to $33 \mathrm{msec}$ ).

The experimental trials were presented at the new display duration. After 84 of the 168 trials, the participants were allowed a brief break. Sessions 2 and 3 began with the display duration set to the last value from the preceding session. The display duration was once again calibrated on the basis of the 30 practice trials, and the remainder of the session was conducted with that display duration.

The location of the displaced letter was cued on 120 of the trials and uncued on the remaining 48 . Of the cued trials, 96 were validly cued, and 24 were invalidly cued. The cues, close, medium, or distant, when present, appeared at the start of each trial for 2,000 msec. Then the grid of three concentric octagons was presented for 2,000 msec. This grid subtended $6^{\circ}$ of visual angle. The stimuli were presented, and then a pattern mask covered the screen for $150 \mathrm{msec}$. The par- 
ticipants then spoke aloud the name of the center letter and the number of the location of the peripheral letter, and the experimenter entered their answers on the keyboard.

\section{RESULTS}

\section{Center Letter}

Mean accuracies for naming the center letter were submitted to an analysis of variance (ANOVA), with span (high or low) as a between-subjects variable and session (first, second, or third) as a within-subjects variable. Overall, high-span participants were more accurate than low-span ones $\left[F(1,54)=13.72, M S_{\mathrm{e}}=0.018, p<\right.$ $.001]$, performance improved over sessions $[F(2,54)=$ $\left.4.21, M S_{\mathrm{e}}=0.088, p<.02\right]$, and the variables interacted $\left[F(2,54)=3.74, M S_{\mathrm{e}}=0.308, p<.03\right]$. This interaction was due to the low-span group's poor performance in Session 1 , in which their mean accuracy was .83 . Mean accuracy for the high-span group in Session 1 was .91. Because of these group differences in accuracy during Session 1, the data from Session 1 were excluded from further analysis. Unless the center letter was accurately identified, one could not be certain that the participant was looking at the fixation point. Mean accuracies for naming the center letter were submitted to an ANOVA with span (high or low) as a between-subjects variable and session (second or third), cue type (valid, invalid, or uncued), and eccentricity of the displaced letter (close, medium, or distant) as within-subjects variables. The ANOVA showed no main effects or interactions. By excluding Session 1, span differences in naming the center letter were eliminated $($ low $=.89$, high $=.91)$, and none of the manipulations on the displaced letter had an effect on naming the center letter.

\section{Display Duration}

Mean display durations for Sessions 2 and 3 were 39.78 and $39.60 \mathrm{msec}$ for the high-span participants and 44.74 and $44.40 \mathrm{msec}$ for the low-span participants. The means were submitted to an ANOVA with span (high or low) as a between-subjects variable and session (second or third) as a within-subjects variable. The ANOVA showed that display times for high-span participants had to be reduced relative to display times for the low-span participants in order to equate their accuracy on naming the center letter $\left[F(1,18)=4.82, M S_{\mathrm{e}}=46.827, p<.04\right]$. There were no other effects.

\section{Localization of the Displaced Letter}

In order to be certain that the participant was focused on the center letter, only the trials in which the center letter was accurately identified were analyzed. In order to evaluate potential differences in the benefit of focusing attention as a function of WM span, the data were first examined in terms of validly cued versus uncued trials. Mean accuracies were submitted to an ANOVA with span (high or low) as a between-subjects variable and eccentricity (close, medium, or distant) and cue type (valid cue or uncued) as within-subjects variables. There was a main effect of eccentricity $\left[F(2,108)=24.79, M S_{\mathrm{e}}=\right.$ $0.036]$. Validly cued trials were superior to uncued trials $\left[F(1,108)=7.88, M S_{\mathrm{e}}=0.076\right]$, as would be expected. Importantly, as can be seen in Table 1, there was no main effect of group $\left[F(1,108)=0.78, M S_{\mathrm{e}}=0.46\right]$, and no interactions were reliable. The procedure that adjusted thresholds for each individual was apparently successful in equating performance for the two groups on the nocue and the validly cued trials (which together were 85\% of the trials). These results suggest that the eccentricity of the displaced letter did not differentially affect high and low WMC individuals. Although eccentricity had an effect on accuracy, more important is the fact that the highand the low-WM participants were similarly affected. This stands in contrast to their dissimilar performances in the invalidly cued condition, to which we now turn.

The question of interest was whether there would be differences in performance on the invalidly cued trials as a function of WM span. As can be seen in Table 2, highspan, but not low-span, participants in the invalidly cued condition showed poorer location of the displaced letter when it occurred on a ring closer to fixation than (i.e., inside) the one cued. This was confirmed by an ANOVA with span (high or low) as a between-subjects variable and location (inside the cued ring or outside the cued ring) as a within-subjects variable. The ANOVA showed no effect of group $\left[F(1,18)<1, M S_{\mathrm{e}}=0.006\right]$ or location $\left[F(1,36)=2.11, M S_{\mathrm{e}}=0.006, p>.10\right]$ but, importantly, did show a group $\times$ location interaction $[F(1,18)=4.37$, $\left.M S_{\mathrm{e}}=0.006, p<.05\right]$. Tests of cell means, which used the $M S_{\mathrm{e}}$ from the associated $F$ test (Toothaker, 1993), showed that location mattered for the low-span participants, whose performance inside the spotlight was superior to that of the high-span participants $[t(18)=2.83$, $p<.011]$ and superior to their performance outside of the cued ring $[t(18)=2.07, p<.05]$. For the high-span

Table 1

Mean Proportions of Correctly Located Displaced Letters by Span Group and Distance From Fixation for Validly Cued and Uncued Trials

\begin{tabular}{|c|c|c|c|c|c|c|c|c|c|c|c|c|}
\hline \multirow{3}{*}{$\begin{array}{c}\text { Working } \\
\text { Memory } \\
\text { Span }\end{array}$} & \multicolumn{6}{|c|}{ Validly Cued Trials } & \multicolumn{6}{|c|}{ Uncued Trials } \\
\hline & \multicolumn{2}{|c|}{ Close } & \multicolumn{2}{|c|}{ Medium } & \multicolumn{2}{|c|}{ Distant } & \multicolumn{2}{|c|}{ Close } & \multicolumn{2}{|c|}{ Medium } & \multicolumn{2}{|c|}{ Distant } \\
\hline & $M$ & SEM & $M$ & $S E M$ & $M$ & $S E M$ & $M$ & SEM & $M$ & $\overline{S E M}$ & $M$ & $\overline{S E M}$ \\
\hline High & .872 & .047 & .907 & .044 & .635 & .048 & .700 & .049 & .906 & .043 & .548 & .047 \\
\hline Low & .759 & .046 & .868 & .045 & .687 & .047 & .658 & .046 & .796 & .047 & .652 & .049 \\
\hline
\end{tabular}


Table 2

Mean Proportions of Correctly Located Displaced Letters by Span Group and Whether the Displaced Letter Occurred Inside or Outside the Cued Location

\begin{tabular}{lccccc}
\hline Working & \multicolumn{2}{c}{ Inside the Cued Ring } & & \multicolumn{2}{c}{ Outside the Cued Ring } \\
\cline { 2 - 3 } \cline { 5 - 6 } Memory & $M$ & $S E M$ & & $M$ & \multicolumn{2}{c}{$S E M$} \\
\hline Span & .664 & .009 & .666 & .031 \\
High & .715 & .015 & .641 & .032 \\
Low & &
\end{tabular}

participants, performance inside the cued ring was comparable to performance outside the cued ring $[t(18)=$ $0.003, p \oplus 1]$.

Not only was low-span performance inside the cued ring superior to performance outside the cued ring, it was equivalent to performance on the validly cued trials. Student's correlated $t$ tests were used to compare other conditions with each group's validly cued condition. When the displaced letter occurred on a more distant ring than the cued ring, both the low-span $[t(9)=2.73, S E=.0311$, $p<.023]$ and the high-span $[t(9)=3.00, S E=.0328, p<$ $.015]$ participants located fewer letters correctly than on their respective validly cued trials. For displaced letters closer to fixation than the cue indicated, the high-span participants performed poorly, as compared with their validly cued performance $[t(9)=3.84, S E=.0263, p<$ .004], replicating Egly and Homa's (1984) findings of flexible allocation and suggesting that the high-span participants were able to focus on the cued ring and ignore the uncued rings. The low-span participants, however, showed no invalid-cue costs when the letter was closer to fixation than the cue indicated $[t(9)=0.84, S E=.0135$, $p>.10]$. This pattern is suggestive of an attentional spotlight such as that suggested by Posner et al. (1980).

\section{CONCLUSIONS}

Individuals with high WM spans differed from those with low WM spans in the way in which they allocated attention. The prediction derived from the controlled attention view of WM was supported: Low WM span individuals were less flexible in their allocation of attention than were their high-span counterparts. The study suggests, more specifically, that low-span individuals tend to allocate their attention much as the original spotlight metaphor of Posner et al. (1980) suggested. On the other hand, high-span individuals were able to allocate their attention to discontiguous locations, the center letter and the cued ring. The possibility of incidental processing of the rings by low-WMC participants is not inconsistent with our assumption of lesser control on their part.

An alternative and equally plausible explanation is that the differences observed here between high- and low-WMC participants resulted from differences in the ability to inhibit the uncued rings (Conway \& Engle, 1994; Hasher \& Zacks, 1988). By this view, high-WMC participants are able to suppress information in the un- cued rings, whereas low-WMC participants are less capable of doing so. It is a very fine distinction between a view arguing that high-WMC participants focus their attention on the cued rings and a view arguing that they suppress information to the uncued rings, and the study presented here was not designed to address this question.

Treating WM as controlled attention led to the prediction confirmed in the present experiment. We believe that the controlled attention view not only has heuristic value, but also will ultimately be shown to capture a fundamental understanding of WM and attention. Our results add to the growing body of literature supporting the equating of WM and controlled attention. Without such an understanding of WM, it is difficult to explain why a dual-task device such as OSPAN predicts the shape in which visual attention can be allocated. Although it may be premature to expect a consensus that WM is controlled attention, the present results do show that WM has an impact on the relatively low-level process of allocating visual attention.

\section{REFERENCES}

Awh, E., \& Pashler, H. (2000). Evidence for split attentional foci. Journal of Experimental Psychology: Human Perception \& Performance, 26, 834-846.

BADDELEY, A. D. (1993). Working memory or working attention. In A. D. Baddeley \& L. Weiskrantz (Eds.), Attention: Selection, awareness, and control. A tribute to Donald Broadbent (pp. 152-170). Oxford: Oxford University Press, Clarendon Press.

BADDEley, A. D., \& Hitch, G. J. (1994). Developments in the concept of working memory. Neuropsychology, 8, 485-493.

CAstiello, U., \& Umiltà, C. (1992). Splitting focal attention. Journal of Experimental Psychology: Human Perception \& Performance, 18, 837-848.

CAVE, K. R., \& Віснот, N. P. (1999). Visuospatial attention: Beyond a spotlight model. Psychonomic Bulletin \& Review, 6, 204-223.

Ce Peda, N. J., Cave, K. R., Bichot, N. P., \& Kim, M.-S. (1998). Spatial selection via feature-driven inhibition of distractor locations. Perception \& Psychophysics, 60, 727-746.

Clarkson-Smith, L., \& Hartley, A. A. (1990). The game of bridge as an exercise in working memory and reasoning. Journals of Gerontology, 45, P233-P238.

CONWAY, A. R. A., CowAn, N., \& Bunting, M. F. (2001). The cocktail party phenomenon revisited: The importance of working memory capacity. Psychonomic Bulletin \& Review, 8, 331-335.

Conway, A. R. A., \& Engle, R. W. (1994). Working memory and retrieval: A resource-dependent inhibition model. Journal of Experimental Psychology: General, 123, 354-373.

Cowan, N. (1999). An embedded-processes model of working memory. In A. Miyake \& P. Shah (Eds.), Models of working memory: Mechanisms of active maintenance and executive control (pp. 62101). New York: Cambridge University Press.

CowAN, N. (2001). The magical number 4 in short-term memory: A reconsideration of mental storage capacity, Behavioral \& Brain Science, 24, 87-185.

DANEMAN, M., \& CARPENTER, P. A. (1980). Individual differences in working memory and reading. Journal of Verbal Learning \& Verbal Behavior, 19, 450-466.

EGLY, R., \& Homa, D. (1984). Sensitization in the visual field. Journal of Experimental Psychology: Human Perception \& Performance, 10, 778-793.

Engle, R. W., KAne, M. J., \& Tuholski, S. W. (1999). Individual differences in working memory capacity and what they tell us about controlled attention, general fluid intelligence, and functions of the 
prefrontal cortex. In A. Miyake \& P. Shah (Eds.), Models of working memory: Mechanisms of active maintenance and executive control (pp. 102-134). New York: Cambridge University Press.

Engle, R. W., Tuholski, S. W., Laughlin, J. E., \& Conway, A. R. A. (1999). Working memory, short-term memory, and general fluid intelligence: A latent-variable approach. Journal of Experimental Psychology: General, 128, 309-331.

HASHER, L., \& ZACKS, R. T. (1988). Working memory, comprehension, and aging: A review and a new view. In G. H. Bower (Ed.), The psychology of learning and motivation: Advances in research and theory (Vol. 22, pp. 193-225). San Diego: Academic Press.

Kane, M. J., Bleckley, M. K., Conway, A. R. A., \& Engle, R. W. (2001). A controlled attention view of working memory capacity. Journal of Experimental Psychology: General, 130, 169-183.

KIEWRA, K. A., \& BEnTON, S. L. (1988). The relationship between information-processing ability and notetaking. Contemporary Educational Psychology, 13, 33-44.

Kramer, A. F., \& HAHn, S. (1995). Splitting the beam: Distribution of attention over noncontiguous regions of the visual field. Psychological Science, 6, 381-386.

Kyllonen, P. C., \& Christal, R. E. (1990). Reasoning ability is (little more than) working-memory capacity? Intelligence, 14, 389-433.
LABerge, D. (1983). Spatial extent of attention to letters and words. Journal of Experimental Psychology: Human Perception \& Performance, 9, 371-379.

Miller, G. A. (1956). The magical number seven plus or minus two: Some limits on our capacity for processing information. Psychological Review, 63, 81-96.

Moray, N. (1959). Attention in dichotic listening: Affective cues and the influence of instructions. Quarterly Journal of Experimental Psychology, 11, 56-60.

Posner, M. I., Snyder, C. R. R., \& Davidson, B. J. (1980). Attention and the detection of signals. Journal of Experimental Psychology: General, 109, 160-174.

SHute, V. J. (1991). Who is likely to acquire programming skills? Journal of Educational Computing Research, 7, 1-24.

TOOTHAKER, L. E. (1993). Quantitative applications in the social sciences: Multiple comparison procedures. Newbury Park, CA: Sage.

TURNER, M., \& ENGLE, R. W. (1989). Is working memory capacity task dependent? Journal of Memory \& Language, 28, 127-154.

(Manuscript received November 7, 2000; revision accepted for publication December 11, 2002.) 